\title{
Adiponectin and Atherosclerosis in Rheumatoid Arthritis
}

\author{
Patrick H. Dessein, ${ }^{1}$ Linda Tsang, ${ }^{1}$ Ahmed Solomon, ${ }^{2}$ Angela J. Woodiwiss, ${ }^{2}$ \\ Aletta M. E. Millen, ${ }^{1}$ and Gavin R. Norton ${ }^{1}$ \\ ${ }^{1}$ Cardiovascular Pathophysiology and Genomics Research Unit, School of Physiology, Faculty of Health Sciences, \\ University of the Witwatersrand, Johannesburg 2193, South Africa \\ ${ }^{2}$ Department of Rheumatology, Charlotte Maxeke Johannesburg Academic Hospital, Faculty of Health Sciences, \\ University of the Witwatersrand, Johannesburg 2193, South Africa
}

Correspondence should be addressed to Patrick H. Dessein; dessein@telkomsa.net

Received 1 March 2014; Accepted 23 May 2014; Published 4 June 2014

Academic Editor: Eeva Moilanen

Copyright (C) 2014 Patrick H. Dessein et al. This is an open access article distributed under the Creative Commons Attribution License, which permits unrestricted use, distribution, and reproduction in any medium, provided the original work is properly cited.

In the present study, we examined the potential impact of adiponectin on carotid ultrasound determined atherosclerosis in 210 (119 black and 91 white) RA patients in mixed regression models. Total adiponectin concentrations were smaller in patients with compared to those without the metabolic syndrome (MetS) defined waist criterion (median (range) $=6.47(1.23-34.54)$ versus 8.38 $(0.82-85.30) \mathrm{ng} / \mathrm{mL}, P=0.02$, resp.); both total and high molecular weight (HMW) adiponectin concentrations were larger in patients with compared to those without joint deformities (7.97 (0.82-85.30) and $3.51(0.01-35.40)$ versus 5.36 (1.29-19.49) and 2.34 (0.01-19.49) ng/mL, $P=0.003$ and 0.02, resp.). Total and HMW adiponectin concentrations were associated with carotid artery plaque in patients with MetS waist (odds ratio $(95 \% \mathrm{CI})=0.87(0.76-0.99)$ and $0.92(0.85-0.99)$ per 1-standard deviation increment, $P=0.02$ for both) and those without joint deformities (odds ratio $(95 \% \mathrm{CI})=0.94(0.88-0.99)$ and $0.94(0.89-0.99), P=0.03$ for both). Plaque prevalence was lower in patients without compared to those with joint deformities ( $23.4 \%$ versus $42.6, P=0.004$ in multivariable analysis). In RA patients with abdominal obesity or no clinically evident joint damage, adiponectin concentrations are reduced but nevertheless associated with decreased carotid atherosclerosis.

\section{Introduction}

Rheumatoid arthritis (RA) increases the risk of atherosclerotic cardiovascular disease (CVD) to a similar extent as diabetes [1-3]. Atherogenesis in RA remains poorly elucidated and current recommendations on CVD risk stratification [4] reportedly have major shortcomings [5, 6]. Hence, there is a need for identifying novel biomarkers of enhanced cardiovascular risk in RA $[5,6]$.

Adiponectin is an adipo(cyto)kine that was identified in 1999 [7]. Its production is paradoxically decreased in obesity [7, 8]. Numerous studies have documented that adiponectin exerts antidiabetic and vasculoprotective effects [8-14]. Amongst the different adiponectin isoforms, high- molecular weight adiponectin particularly protects against the development of diabetes [15] and CVD [16].

In cellular studies, adiponectin was shown to increase gene expression and protein synthesis of many proinflammatory and prodestructive molecules that participate in the pathophysiology of RA [17-19].

Both the production and effects of adipokines can be altered in patients with autoimmune diseases including RA $[20,21]$. In this regard, whether adiponectin protects against CVD in RA is currently uncertain. Indeed, increased as well as reduced adiponectin concentrations in RA were reported by different groups [22-24]. Also both inverse or favorable and direct or paradoxically unfavorable associations of adiponectin concentrations with metabolic risk factors 
were found in RA [21, 24, 25]. With regard to direct effects of adiponectin on atherogenesis, we recently reported a cardiovascular risk factor independent direct relationship between adiponectin concentrations and early endothelial activation in white patients with RA [25]. Additionally, no association was found between adiponectin levels as well as carotid intima-media thickness (cIMT) and coronary artery calcification scores in RA [21, 26, 27]. However, compared to cIMT, carotid plaque is a more reliable indicator of severe atherosclerosis [28-34]. Thus, cIMT reflects mostly arterial media thickening in response to aging and elevated blood pressure whereas plaque represents intimal pathology and advanced atherosclerosis that links more closely to coronary heart disease risk factors and myocardial infarction [28-34].

In the present study, we examined the independent relationships of total and HMW adiponectin concentrations with cIMT and plaque. As the production and effects of adipokines on cardiovascular risk depend on pathophysiological context [21, 25, 35-39], we also determined whether the presence of conventional and nonconventional cardiovascular risk factors modified adiponectin concentrations and their associations with atherosclerosis.

\section{Patients and Methods}

2.1. Patients. The present study was conducted according to the principles outlined in the Helsinki declaration. The Human Research Ethics Committee (Medical) from the University of the Witwatersrand in Johannesburg, South Africa, approved the protocol (approval number: M06-07-33). Participants gave informed, written consent. This investigation forms part of an ongoing study on cardiovascular risk in RA $[25,36,38,39]$. Two hundred and ten consecutive African patients (119 black and 91 white) that met the 1988 American College of Rheumatology and 2010 American College of Rheumatology/EULAR criteria for RA [40, 41] were enrolled. Carotid ultrasound was performed in all except 2 white patients. All invited participants agreed to participate. Data were missing in fewer than $5 \%$ of any of the recorded characteristics.

2.2. Assessments. Baseline characteristics and conventional metabolic risk factors were recorded using previously reported methods [25, 36, 38, 39]. Briefly, we recorded demographic features and lifestyle factors. Height, weight, and waist and hip circumference were measured using standard approaches. Overall adiposity, abdominal obesity, and fat distribution were estimated by the body mass index (BMI), waist circumference, and waist-hip ratio, respectively [28]. We recorded disease duration and rheumatoid factor status. Disease activity was assessed by the Clinical Disease Activity Index (CDAI) [42]. Extra-articular manifestations included the current or previously recorded (hospital record review) presence of pericarditis, pleuritis, Felty's syndrome, cutaneous vasculitis, neuropathy, scleritis or episcleritis, retinal vasculitis, glomerulonephritis, vasculitis affecting other organs, amyloidosis, keratoconjunctivitis sicca, xerostomia,
Sjogren's syndrome, pulmonary fibrosis, bronchiolitis obliterans organizing pneumonia, cervical myelopathy, subcutaneous nodules, and rheumatoid nodules in other locations [43]. C-reactive protein concentrations were determined using immunoturbidimetric methods. Standard laboratory blood tests of erythrocyte sedimentation rate, renal and liver function, hematological parameters, lipids, and glucose were performed. The glomerular filtration rate was estimated using the Modification of Diet in Renal Disease equation [44]. Cardiovascular drug use was recorded.

Recorded metabolic risk factors included systolic, diastolic and mean blood pressure, lipid concentrations and ratios, and glucose levels. Hypertension was defined as an average systolic blood pressure $\geq 140$ or/and diastolic blood pressure $\geq 90 \mathrm{mmHg}$ or/and current use of antihypertensive medications. Dyslipidemia was diagnosed when the atherogenic index, that is, the cholesterol-HDL cholesterol ratio, was $>4$ [45]. Diabetes was identified as the use of glucose lowering agents or a fasting plasma glucose $\geq 7 \mathrm{mmol} / \mathrm{L}$.

As reported previously, we also measured resistin concentrations [36].

BAS (see acknowledgement) and AS performed the carotid artery ultrasound measurements in private and public healthcare patients, respectively. Both operators obtained images of at least $1 \mathrm{~cm}$ length of the distal common carotid arteries for measurement of the intima-media thickness of the far wall from an optimal angle of incidence defined as the longitudinal angle of approach where both branches of the internal and external carotid artery are visualized simultaneously [46] and with high resolution B-mode ultrasound (Image Point, Hewlett Packard, Andover, MA, USA, and SonoCalc IMT, Sonosite Inc., Bothell, Wash, USA, used by BAS and AS, resp.) employing linear array $7.5 \mathrm{MHz}$ probes. The details of the methodology used by BAS were reported previously [47]. The equipment used by AS involves the application of a unique semiautomated border detection program that was previously found to provide highly reproducible results [46]. The intima-media thicknesses in the left and right common carotid artery were measured and the cIMT was defined as the mean of of the two obtained values. Carotid artery plaque was defined as a focal structure that encroaches into the arterial lumen of at least $0.5 \mathrm{~mm}$ or $50 \%$ of the surrounding intima-media thickness value or demonstrates a thickness of $>1.5 \mathrm{~mm}$ as measured from the media-adventitia interface to the intima-lumen interface [48]. Both operators were blinded to the cardiovascular risk profiles of the patients. Repeat ultrasound examinations by both operators on 23 patients revealed Spearman correlations between repeat cIMT measurements of 0.983 and 0.956 for BAS and AS, respectively, and the correlation between measurements made by BAS and AS was 0.926 . Both operators identified carotid artery bulb or/and internal carotid artery plaque in 11 of these 23 patients with full agreement.

Total and HMW adiponectin concentrations were measured using solid-phase sandwich enzyme-linked immunosorbent assays (ELISA) (Quantikine HS, R\&D Systems, Inc., Minneapolis, MN, USA). Their lower detection limits were 0.246 and $0.195 \mathrm{ng} / \mathrm{mL}$, respectively. The inter- and intra- 
TABLE 1: Total and high molecular weight adiponectin in all 210 RA patients and subgroups.

\begin{tabular}{|c|c|c|c|c|c|}
\hline \multirow[t]{2}{*}{ Groups } & \multirow[t]{2}{*}{ Number } & \multicolumn{2}{|c|}{$\begin{array}{c}\text { Total adiponectin } \\
(\mathrm{ng} / \mathrm{mL})\end{array}$} & \multicolumn{2}{|c|}{$\begin{array}{c}\text { HMW adiponectin } \\
(\mathrm{ng} / \mathrm{mL})\end{array}$} \\
\hline & & Median (IQR) & $P$ & Median (IQR) & $P$ \\
\hline All & 210 & $7.41(4.89-11.97)$ & - & $3.26(1.27-5.74)$ & - \\
\hline \multicolumn{6}{|l|}{ Population } \\
\hline Black & 119 & $7.41(5.62-11.56)$ & & $2.65(1.55-5.53)$ & \\
\hline White & 91 & $7.25(5.31-12.83)$ & 0.9 & $3.82(2.10-6.00)$ & 0.9 \\
\hline \multicolumn{6}{|c|}{ Age $>55$ years } \\
\hline Yes & 122 & $7.45(5.20-13.58)$ & & $3.58(1.86-6.18)$ & \\
\hline No & 88 & $6.84(4.67-10.13)$ & 0.2 & $2.67(1.38-4.76)$ & 0.1 \\
\hline \multicolumn{6}{|c|}{$\geq 2$ major risk factors } \\
\hline Yes & 146 & $7.17(4.62-11.39)$ & & $2.99(1.53-5.40)$ & \\
\hline No & 55 & $8.48(5.90-13.28)$ & 0.1 & $3.78(2.07-6.32)$ & 1.0 \\
\hline Missing & 9 & & & & \\
\hline \multicolumn{6}{|l|}{ Obesity } \\
\hline Yes & 64 & $6.91(4.32-9.76)$ & & $2.56(1.26-4.96)$ & \\
\hline No & 140 & $7.58(5.28-13.23)$ & 0.7 & $3.47(2.00-6.09)$ & 0.9 \\
\hline Missing & 6 & & & & \\
\hline \multicolumn{6}{|l|}{ MetS waist } \\
\hline Yes & 100 & $6.47(4.32-10.01)$ & & $2.66(1.42-4.76)$ & \\
\hline No & 107 & $8.38(5.32-13.63)$ & 0.02 & $3.57(2.03-6.48)$ & 0.3 \\
\hline Missing & 3 & & & & \\
\hline \multicolumn{6}{|c|}{ RA duration $>10$ years } \\
\hline Yes & 109 & $7.41(4.99-12.96)$ & & $3.44(1.74-6.13)$ & \\
\hline No & 100 & $7.32(4.32-11.29)$ & 0.5 & $2.80(1.53-4.94)$ & 0.9 \\
\hline Missing & 1 & & & & \\
\hline \multicolumn{6}{|l|}{$\mathrm{CDAI}>10$} \\
\hline Yes & 93 & $7.51(4.65-11.94)$ & & $3.33(1.57-5.69)$ & \\
\hline No & 116 & $7.35(5.04-12.83)$ & 0.4 & $3.26(1.98-5.87)$ & 0.8 \\
\hline Missing & 1 & & & & \\
\hline \multicolumn{6}{|c|}{ ESR $>12 \mathrm{~mm} / \mathrm{hr}$} \\
\hline Yes & 116 & $7.38(4.50-11.29)$ & & $3.29(1.69-5.60)$ & \\
\hline No & 87 & $7.87(5.31-12.92)$ & 0.4 & $3.26(1.71-5.90)$ & 0.8 \\
\hline Missing & 7 & & & & \\
\hline \multicolumn{6}{|c|}{ Deformed joints } \\
\hline Yes & 162 & $7.97(5.19-13.58)$ & & $3.51(1.81-6.00)$ & \\
\hline No & 47 & $5.36(3.55-8.56)$ & 0.0003 & $2.34(1.38-4.14)$ & 0.02 \\
\hline Missing & 1 & & & & \\
\hline \multicolumn{6}{|l|}{ RF positive } \\
\hline Yes & 161 & $7.41(4.90-11.23)$ & & $3.27(1.78-5.58)$ & \\
\hline No & 48 & $7.51(3.95-15.03)$ & 0.7 & $2.74(1.29-6.49)$ & 0.6 \\
\hline Missing & 1 & & & & \\
\hline
\end{tabular}

Associations were identified in age, sex, race, glomerular filtration rate, cardiovascular drug use, and waist circumference adjusted models. Significant associations are shown in bold. RA: rheumatoid arthritis; HMW: high molecular weight; IQR: interquartile range; MetS: metabolic syndrome; CDAI: Clinical Disease Activity Index; ESR: erythrocyte sedimentation rate; RF: rheumatoid factor.

assay coefficients of variation were 6.5 and $3.5 \%$ for total and 8.5 and $3.0 \%$ for high molecular weight adiponectin, respectively.

2.3. Data Management Analysis. Dichotomous variables are expressed as proportions or percentages and continuous variables as mean (SD) or median (interquartile range (IQR)) when nonnormally distributed.

Associations of baseline characteristics with total and HMW adiponectin concentrations in the present cohort were previously reported and considered in multivariable analysis in the present study, that is, upon deciding which characteristics are potential confounders or mediators. These variables 
TABLE 2: Carotid atherosclerosis in 208 patients with rheumatoid arthritis.

\begin{tabular}{lc}
\hline Intima-media thickness, $\mathrm{mm}$ & $0.710(0.108)$ \\
Plaque & 38.1 \\
\hline
\end{tabular}

Continuous variable expressed as mean (SD) and categorical variable as proportion.

comprised age, sex, race, glomerular filtration rate, and waist circumference. We assessed the associations of HMW and total adiponectin concentrations with cIMT and plaque in Framingham score (calculated from age, sex, and major conventional risk factors), race, glomerular filtration rate, waist circumference, and C-reactive protein level adjusted mixed linear or logistic regression models as appropriate. The Framingham score was used rather than its individual components in order to avoid over-fitted models as these can produce false positive and false negative results.

Patients with RA that experience conventional risk factors or severe disease are reportedly at high risk of cardiovascular disease [1-6]. For these reasons together with the influence of physiological context on adipokine effects and our recent experience with adipokine metabolism in RA [21, 25, 3539], we assessed the impact of patient characteristics on total and HMW adiponectin concentrations and their relations with carotid cIMT and plaque in subgroups with and without patient characteristics of interest in the present context. For this purpose, patients with a BMI of $\geq 30 \mathrm{~kg} / \mathrm{m}^{2}$ and those that met the National Cholesterol Education Program for metabolic syndrome (MetS) waist criterion [49] were considered to sustain overall and abdominal obesity, respectively. When appropriate, patients were categorized in subgroups based on median values. In view of the small number of patients that were rheumatoid factor negative or had extraarticular features, sensitivity analysis in subgroups based on the presence or absence of these characteristics was not performed.

Statistical computations were made using the GB Stat program (Dynamic Microsystems, Inc., Silverspring, Maryland, USA) and SAS software, version 9.1 (The SAS Institute, Cary, NC). Significance was set at $P$ value $\leq 0.05$.

\section{Results}

3.1. Patient Characteristics. The demographic features, lifestyle factors, anthropometric measures, conventional metabolic risk factors, C-reactive protein concentrations, glomerular filtration rate, and use of antirheumatic and cardiovascular agents in the present cohort were previously reported [25]. The median (range) erythrocyte sedimentation rate was $14(0-120) \mathrm{mm} / \mathrm{hr}$.

Total and HMW adiponectin concentrations in all patients and relevant subgroups are given in Table 1. Total adiponectin concentrations were smaller in patients with compared to those without abdominal obesity and both total and HMW adiponectin concentrations larger in those with compared to those without deformed joints.
The mean (SD) cIMT and plaque prevalence in 208 of the patients is given in Table 2 .

3.2. Independent Associations of Total and HMW Adiponectin Concentrations with Atherosclerosis. The relationships between total adiponectin and CIMT and plaque are shown in Table 3. Total adiponectin concentrations were not associated with atherosclerosis in all patients. However, total adiponectin concentrations were related to plaque prevalence in patients with but not without abdominal obesity and in those without but not with joint deformities.

The relationships between HMW adiponectin and cIMT and plaque are shown in Table 4 . As applied to total adiponectin, HMW adiponectin concentrations were not associated with atherosclerosis in all patients and related to plaque prevalence in patients with but not without abdominal obesity and in those without but not with joint deformities.

The use of the Framingham score in our analysis may not have fully accounted for the confounding effect of age on atherosclerosis. However, in additional analysis in which we constructed models in groups as in Tables 4 and 5 but with adjustment for age, sex, race, and waist circumference as potential confounders, total and HMW adiponectin concentrations remained associated with plaque in patients with abdominal obesity and without joint deformities $(P=0.02$ for each relationship).

In view of these findings we evaluated whether cIMT and plaque prevalence differed by abdominal obesity and joint deformity status. The respective results are given in Table 5 . Plaque prevalence was numerically smaller in patients with compared to those without abdominal obesity and larger in patients with compared to those without joint deformities. The latter relationship was significant with an odds ratio $(95 \%$ confidence interval) of 3.31 (1.46-7.51) for the association of deformed joint with plaque. In this regard also, obesity protects against joint damage in RA [50]. In the present study, the median (range) deformed joint count was smaller in patients with compared to those without abdominal obesity (5 (0-36) versus $10(0-45), P=0.05)$.

When, in additional analysis, we constructed models in groups as in Table 5 but with adjustment for age, sex, race, and waist circumference as potential confounders, the plaque prevalence remained higher in patients with compared to those without deformed joints $(P=0.01)$.

3.3. Independent Associations of Total and HMW Adiponectin Concentrations with Those of Resistin. In age, sex, race, glomerular filtration rate, cardiovascular drug use, and waist circumference adjusted models, total and HMW adiponectin concentrations were not significantly $(P \geq 0.2)$ associated with those of resistin in all patients and those with and without abdominal obesity or deformed joints. Also, upon further adjustment for resistin concentrations in Tables 3 and 4, total and HMW adiponectin concentrations remained associated with plaque ( $P=0.02$ to 0.002$)$ in patients with abdominal obesity and without joint deformities.

Several recorded characteristics were nonnormally distributed in the present investigation. When we repeated 
TABLE 3: Independent relationships of total adiponectin concentrations (1 SD increment) with carotid atherosclerosis in all RA patients and subgroups.

\begin{tabular}{|c|c|c|c|c|}
\hline \multirow{2}{*}{ Groups } & \multicolumn{2}{|c|}{ cIMT } & \multicolumn{2}{|c|}{ Plaque } \\
\hline & Partial $R$ & $P$ & OR $(95 \% \mathrm{CI})$ & $P$ \\
\hline All & 0.042 & 0.6 & $0.98(0.91-1.05)$ & 0.6 \\
\hline \multicolumn{5}{|c|}{ Population } \\
\hline Black & -0.153 & 0.1 & $1.08(0.94-1.30)$ & 0.9 \\
\hline White & 0.131 & 0.2 & $0.89(0.77-1.01)$ & 0.07 \\
\hline \multicolumn{5}{|c|}{ Age $>55$ years } \\
\hline Yes & 0.031 & 0.8 & $0.98(0.89-1.07)$ & 0.07 \\
\hline No & 0.023 & 0.8 & $0.92(0.75-1.02)$ & 0.08 \\
\hline \multicolumn{5}{|c|}{$\geq 1$ major risk factors } \\
\hline Yes & -0.023 & 0.9 & $0.95(0.89-1.01)$ & 0.09 \\
\hline No & 0.041 & 0.6 & $1.05(0.90-1.02)$ & 0.5 \\
\hline \multicolumn{5}{|l|}{ Obesity } \\
\hline Yes & 0.068 & 0.6 & $0.93(0.84-1.02)$ & 0.1 \\
\hline No & 0.040 & 0.7 & $0.99(0.90-1.09)$ & 0.9 \\
\hline \multicolumn{5}{|c|}{ MetS waist } \\
\hline Yes & 0.112 & 0.3 & $0.87(0.76-0.99)$ & 0.02 \\
\hline No & 0.003 & 1.0 & $1.08(0.92-1.13)$ & 0.7 \\
\hline \multicolumn{5}{|c|}{ RA duration $>10$ years } \\
\hline Yes & 0.129 & 0.2 & $0.94(0.83-1.03)$ & 0.2 \\
\hline No & -0.078 & 0.5 & $1.05(0.94-1.15)$ & 0.4 \\
\hline \multicolumn{5}{|l|}{$\mathrm{CDAI}>10$} \\
\hline Yes & -0.017 & 0.9 & $0.92(0.78-1.06)$ & 0.2 \\
\hline No & 0.090 & 0.4 & $0.99(0.90-1.08)$ & 0.7 \\
\hline \multicolumn{5}{|l|}{ ESR $>12$} \\
\hline Yes & -0.072 & 0.5 & $0.96(0.84-1.08)$ & 0.4 \\
\hline No & 0.129 & 0.2 & $0.97(0.87-1.08)$ & 0.6 \\
\hline \multicolumn{5}{|c|}{ Deformed joints } \\
\hline Yes & 0.068 & 0.4 & $1.00(0.90-1.10)$ & 0.9 \\
\hline No & -0.193 & 0.2 & $0.94(0.88-0.99)$ & 0.03 \\
\hline \multicolumn{5}{|c|}{ Rheumatoid factor positive } \\
\hline Yes & 0.023 & 0.8 & $0.99(0.94-1.04)$ & 0.9 \\
\hline No & 0.091 & 0.6 & $0.91(0.72-1.10)$ & 0.3 \\
\hline
\end{tabular}

Relationships were determined in Framingham score, race, glomerular filtration rate, waist circumference, and C-reactive protein concentrations adjusted models. Significant associations are shown in bold. SD: standard deviation; cIMT: carotid intima-media thickness; OR: odds ratio; CI: confidence interval; RA: rheumatoid arthritis; MetS: metabolic syndrome; CDAI: Clinical Disease Activity Index; ESR: erythrocyte sedimentation rate.

the analyses using log transformed variables in the mixed regression models, the results were materially unaltered (data not shown).

\section{Discussion}

Previous studies on the potential involvement of adiponectin in enhanced CVD risk in RA have produced contradictory results [24-27]. Herein, we show for the first time that both total and HMW adiponectin concentrations are independently associated with reduced plaque prevalence in RA patients with abdominal obesity or clinical absent joint damage. A 1-standard deviation increment in total adiponectin concentration was independently associated with a 13 and $6 \%$ reduction in plaque prevalence amongst patients with abdominal obesity and clinical absent joint damage, respectively. Carotid artery plaque independently predicts incident cardiovascular event rates in non-RA subjects and patients with RA [51].

Our findings have at least 4 implications. First, as applies to osteoprotegerin [52] and retinol binding protein 4 (RBP4) [38], which is an adipokine that enhances atherogenesis, 
TABLE 4: Independent relationships of high molecular weight adiponectin concentrations (1 SD increment) with carotid atherosclerosis in all RA patients and subgroups.

\begin{tabular}{|c|c|c|c|c|}
\hline \multirow{2}{*}{ Groups } & \multicolumn{2}{|c|}{ cIMT } & \multicolumn{2}{|c|}{ Plaque } \\
\hline & Partial $R$ & $P$ & OR $(95 \% \mathrm{CI})$ & $P$ \\
\hline All & -0.008 & 0.9 & $0.99(0.96-1.02)$ & 0.6 \\
\hline \multicolumn{5}{|c|}{ Population } \\
\hline Black & -0.173 & 0.08 & $1.04(0.94-1.33)$ & 0.9 \\
\hline White & 0.071 & 0.5 & $0.96(0.95-1.01)$ & 0.1 \\
\hline \multicolumn{5}{|c|}{ Age $>55$ years } \\
\hline Yes & -0.057 & 0.6 & $0.99(0.94-1.04)$ & 0.07 \\
\hline No & 0.008 & 0.9 & $0.95(0.91-1.01)$ & 0.1 \\
\hline \multicolumn{5}{|c|}{$\geq 1$ major risk factors } \\
\hline Yes & -0.042 & 0.8 & $0.96(0.92-1.01)$ & 0.07 \\
\hline No & -0.035 & 0.7 & $1.01(0.95-1.12)$ & 0.4 \\
\hline \multicolumn{5}{|l|}{ Obesity } \\
\hline Yes & -0.008 & 1.0 & $0.96(0.90-1.01)$ & 0.1 \\
\hline No & -0.011 & 0.9 & $1.00(0.96-1.05)$ & 1.0 \\
\hline \multicolumn{5}{|c|}{ MetS waist } \\
\hline Yes & -0.035 & 0.7 & $0.92(0.85-0.99)$ & 0.02 \\
\hline No & -0.006 & 1.0 & $1.02(0.94-1.06)$ & 0.6 \\
\hline \multicolumn{5}{|c|}{ RA duration $>10$ years } \\
\hline Yes & 0.059 & 0.6 & $0.97(0.92-1.02)$ & 0.2 \\
\hline No & -0.091 & 0.4 & $1.02(0.97-1.07)$ & 0.5 \\
\hline \multicolumn{5}{|l|}{$\mathrm{CDAI}>10$} \\
\hline Yes & 0.005 & 1.0 & $0.94(0.86-1.02)$ & 0.2 \\
\hline No & -0.002 & 1.0 & $1.00(0.96-1.04)$ & 0.9 \\
\hline \multicolumn{5}{|l|}{ ESR $>12$} \\
\hline Yes & -0.013 & 0.9 & $0.96(0.84-1.08)$ & 0.3 \\
\hline No & 0.038 & 0.7 & $0.99(0.96-1.04)$ & 0.7 \\
\hline \multicolumn{5}{|c|}{ Deformed joints } \\
\hline Yes & 0.018 & 0.8 & $1.00(0.96-1.05)$ & 0.8 \\
\hline No & -0.140 & 0.4 & $0.94(0.89-0.99)$ & 0.03 \\
\hline \multicolumn{5}{|c|}{ Rheumatoid factor positive } \\
\hline Yes & 0.008 & 0.9 & $1.00(0.96-1.03)$ & 0.8 \\
\hline No & -0.024 & 0.9 & $0.96(0.86-1.05)$ & 0.3 \\
\hline
\end{tabular}

Relationships were determined in Framingham score, race, glomerular filtration rate, waist circumference, and C-reactive protein concentrations adjusted models. Significant associations are shown in bold. SD: standard deviation; cIMT: carotid intima-media thickness; OR: odds ratio; CI: confidence interval; RA: rheumatoid arthritis; MetS: metabolic syndrome; CDAI: Clinical Disease Activity Index; ESR: erythrocyte sedimentation rate.

adiponectin is a promising cardiovascular risk biomarker that could improve cardiovascular risk stratification in RA [46]. Second, considering adiponectin concentrations only may be inadequate in estimating the impact of this adipokine in cardiovascular risk in RA. Indeed, despite the presence of reduced total and HMW adiponectin concentrations in patients with abdominal obesity or absent joint damage, it was in these groups that we observed inverseadiponectin atherosclerosis relations. Thus, small rather large adiponectin concentrations concurred with a favorable association with cardiovascular risk in the present investigation. Taken together, increased adiponectin concentrations in RA do not necessarily translate into adiponectin mediated cardioprotective effects. Third, targeting adiponectin in an attempt to reduce RA severity [17-19] could be expected to additionally increase cardiovascular risk, at least in patients with abdominal obesity or less severe RA. Fourth, as previously documented by us [36-39], stratified analysis should be considered upon elucidating of the potential role of adipokines in CVD risk in RA.

Obesity is paradoxically associated with decreased incident cardiovascular event rates [53] and overall mortality in RA [54]. In this study, plaque prevalence was numerically reduced in patients with abdominal obesity. Congruently, we recently also found an independent inverse relation between abdominal adiposity and endothelial activation in RA [55]. 
TABLE 5: Carotid atherosclerosis in RA patients by abdominal obesity and clinical joint damage status.

\begin{tabular}{lccccc}
\hline Subgroups & Number & cIMT, mm & $P$ & Plaque & $P$ \\
\hline MetS waist & & & & & \\
$\quad$ Yes & 100 & $0.709(0.098)$ & & 31.0 & \\
No & 107 & $0.713(0.118)$ & 0.9 & 45.8 & 0.5 \\
$\quad$ Missing & 3 & & & & \\
Deformed joint & & & & & \\
$\quad$ Yes & 162 & $0.709(0.104)$ & & $\mathbf{4 2 . 6}$ & \\
No & 47 & $0.719(0.123)$ & 0.4 & $\mathbf{2 3 . 4}$ & $\mathbf{0 . 0 0 4}$ \\
Missing & 1 & & & & \\
\hline
\end{tabular}

Continuous variables expressed as mean (SD) and categorical variables as proportions. Relationships were identified in Framingham score, race, Creactive protein concentrations, and waist circumference adjusted models. Significant association is shown in bold. RA: rheumatoid arthritis; cIMT: carotid intima-media thickness; MetS: metabolic syndrome.

The present investigation suggests that altered and favorable effects of adiponectin may constitute a mechanism that links abdominal obesity to reduced cardiovascular risk in RA.

Obesity also reportedly protects against joint damage in RA. In one study this relationship was explained by adiponectin concentrations [50]. In the present investigation, we also found an inverse association between abdominal obesity and joint damage. Joint deformity counts as well as radiographic damage in RA are associated with increased atherosclerosis [47]. Our current results further indicate that altered effects of adiponectin may additionally contribute to the link between mild RA and reduced cardiovascular risk.

We recently reported a paradoxically positive relation between adiponectin concentrations and endothelial activation amongst white patients with RA [25]. As also indicated by other investigators [56], we suggested that this finding represents a compensatory increase in adiponectin production aimed at reducing cardiovascular risk rather than a paradoxically altered adverse impact on atherogenesis [25]. Our current results support this possibility since total adiponectin concentrations exhibited a borderline inverse association with plaque prevalence in white patients $(\mathrm{OR}=0.89$ per $1-$ standard deviation increment, $P=0.07$ ). Interestingly in this regard, we recently documented that RBP4 concentrations are paradoxically associated with reduced endothelial activation and, at the same time, also with enhanced atherosclerosis in RA [38].

High grade inflammation is associated with atherosclerosis and cardiovascular events in RA $[57,58]$. In this regard, adiponectin is an anti-inflammatory adipokine [17-19, 21, 25] whereas resistin is proinflammatory $[36,59]$. Indeed, resistin concentrations are associated with those of C-reactive protein in RA $[36,59]$. Moreover, resistin levels are independently related to surrogate markers of early atherogenesis and may contribute to the link between inflammation and enhanced cardiovascular risk in RA [36]. Our analysis argues against circulating adiponectin and resistin being linked in RA and the adiponectin concentration-atherosclerosis relations were not explained by resistin levels.
In a recent investigation, Koskinen and colleagues [60] showed that in men with osteoarthritis (OA), circulating adiponectin concentrations correlate positively with levels of OA biomarkers and both circulating adiponectin levels and adiponectin concentrations released by cultured cartilage are associated with OA severity. Furthermore, when adiponectin was added at physiological concentrations to cultures of intact OA cartilage or primary OA chondrocytes, this adipokine enhanced the production of inflammatory and/or destructive factors nitric oxide, interleukin-6, and matrix metalloproteinases 1 and 3 in a mitogen-activated protein kinase-dependent manner. Hence, reported evidence together with the findings in the current study indicates that adiponectin can exert a dual role in arthritis comprising catabolic and proinflammatory effects on cartilage and protection against atherosclerosis.

We evaluated relationships of both total and HMW adiponectin concentrations with atherosclerosis in a relatively large and well characterized RA cohort. A substantial number of potential confounders or/and mediators were included in mixed regression models. Our study nevertheless has limitations. The cross-sectional design precludes drawing inferences on the direction of causality. We assessed joint damage by joint deformity counts rather than radiographic scores. However, we previously found that radiographic scores and joint deformities are strongly correlated in our setting $(R=0.808, P<0.0001)$ [47]. We did not investigate matched healthy controls. Finally, circulating adiponectin concentrations may not necessarily represent its tissue levels.

In conclusion, the present study indicates that consideration of adiponectin can improve cardiovascular risk stratification amongst RA patients with abdominal obesity or mild disease. These findings merit further investigation in future longitudinal studies.

\section{Conflict of Interests}

The authors declare no interest of interests.

\section{Acknowledgments}

This work is supported by Medical Research Council Grant MRC2008_DES and the National Research Foundation (South Africa).

\section{References}

[1] P. H. Dessein, G. R. Norton, A. J. Woodiwiss, B. I. Joffe, and F. Wolfe, "Influence of nonclassical cardiovascular-risk factors on the accuracy of predicting subclinical atherosclerosis in rheumatoid arthritis," Journal of Rheumatology, vol. 34, no. 5, pp. 943-951, 2007.

[2] D. H. Solomon, J. Kremer, J. R. Curtis et al., "Explaining the cardiovascular risk associated with rheumatoid arthritis: traditional risk factors versus markers of rheumatoid arthritis severity," Annals of the Rheumatic Diseases, vol. 69, no. 11, pp. 1920-1925, 2010.

[3] M. T. Nurmohamed and G. Kitas, "Cardiovascular risk in rheumatoid arthritis and diabetes: how does it compare and 
when does it start?" Annals of the Rheumatic Diseases, vol. 70, no. 6, pp. 881-883, 2010.

[4] M. J. L. Peters, D. P. M. Symmons, D. McCarey et al., "EULAR evidence-based recommendations for cardiovascular risk management in patients with rheumatoid arthritis and other forms of inflammatory arthritis," Annals of the Rheumatic Diseases, vol. 69, no. 2, pp. 325-331, 2010.

[5] C. S. Crowson and S. E. Gabriel, “Towards improving cardiovascular risk management in patients with rheumatoid arthritis: the need for accurate risk assessment," Annals of the Rheumatic Diseases, vol. 70, no. 5, pp. 719-721, 2011.

[6] P. H. Dessein and A. G. Semb, "Could cardiovascular disease risk stratification and management in rheumatoid arthritis be enhanced?" Annals of the Rheumatic Diseases, vol. 72, no. 11, pp. 1743-1746, 2013.

[7] Y. Arita, S. Kihara, N. Ouchi et al., "Paradoxical decrease of an adipose-specific protein, adiponectin, in obesity," Biochemical and Biophysical Research Communications, vol. 257, no. 1, pp. 79-83, 1999.

[8] Y. Yamamoto, H. Hirose, I. Saito et al., "Correlation of the adipocyte-derived protein adiponectin with insulin resistance index and serum high-density lipoprotein-cholesterol, independent of body mass index, in the Japanese population," Clinical Science, vol. 103, no. 2, pp. 137-142, 2002.

[9] A. Shehzad, W. Iqbal, O. Shehzad, and Y. S. Lee, "Adiponectin: regulation of its production and its role in human diseases," Hormones, vol. 11, no. 1, pp. 8-20, 2012.

[10] G. Fantuzzi and T. Mazzone, "Adipose tissue and atherosclerosis: exploring the connection," Arteriosclerosis, Thrombosis, and Vascular Biology, vol. 27, no. 5, pp. 996-1003, 2007.

[11] M. Tanida, J. Shen, Y. Horii et al., "Effects of adiponectin on the renal sympathetic nerve activity and blood pressure in rats," Experimental Biology and Medicine, vol. 232, no. 3, pp. 390-397, 2007.

[12] S. G. Wannamethee, J. Tchernova, P. Whincup et al., "Associations of adiponectin with metabolic and vascular risk parameters in the British Regional Heart Study reveal stronger links to insulin resistance-related than to coronory heart disease riskrelated parameters," International Journal of Obesity, vol. 31, no. 7, pp. 1089-1098, 2007.

[13] Y. Iwashima, T. Katsuya, K. Ishikawa et al., "Hypoadiponectinemia is an independent risk factor for hypertension," Hypertension, vol. 43, no. 6, pp. 1318-1323, 2004.

[14] S. Li, H. J. Shin, E. L. Ding, and R. M. van Dam, "Adiponectin levels and risk of type 2 diabetes: a systematic review and metaanalysis," JAMA-Journal of the American Medical Association, vol. 302, no. 2, pp. 179-188, 2009.

[15] C. Lara-Castro, N. Luo, P. Wallace, R. L. Klein, and W. T. Garvey, "Adiponectin multimeric complexes and the metabolic syndrome trait cluster," Diabetes, vol. 55, no. 1, pp. 249-259, 2006.

[16] H. Kobayashi, N. Ouchi, S. Kihara et al., "Selective suppression of endothelial cell apoptosis by the high molecular weight form of adiponectin," Circulation Research, vol. 94, no. 4, pp. e27-e31, 2004.

[17] R. Krysiak, G. Handzlik-Orlik, and B. Okopien, "The role of adipokines in connective tissue diseases," European Journal of Nutrition, vol. 51, no. 5, pp. 513-528, 2012.
[18] K. Frommer, A. Schäffler, C. Büchler et al., "Adiponectin isoforms: a potential therapeutic target in rheumatoid arthritis?" Annals of the Rheumatic Diseases, vol. 71, no. 10, pp. 1724-1732, 2012.

[19] K. W. Frommer, B. Zimmermann, F. M. P. Meier et al., "Adiponectin-mediated changes in effector cells involved in the pathophysiology of rheumatoid arthritis," Arthritis and Rheumatism, vol. 62, no. 10, pp. 2886-2899, 2010.

[20] B. H. Hahn, E. V. Lourenço, M. McMahon et al., "Pro-inflammatory high-density lipoproteins and atherosclerosis are induced in lupus-prone mice by a high-fat diet and leptin," Lupus, vol. 19, no. 8, pp. 913-917, 2010.

[21] P. H. Dessein, G. R. Norton, M. Badenhorst, A. J. Woodiwiss, and A. Solomon, "Rheumatoid arthritis impacts on the independent relationships between circulating adiponectin concentrations and cardiovascular metabolic risk," Mediators of Inflammation, vol. 2013, Article ID 461849, 9 pages, 2013.

[22] M. Otero, R. Logo, R. Gomez et al., "Changes in plasma levels of fat-derived hormones adiponectin, leptin, resistin and visfatin in patients with rheumatoid arthritis," Annals of the Rheumatic Diseases, vol. 65, no. 9, pp. 1198-1201, 2006.

[23] C. Popa, M. G. Netea, J. de Graaf et al., "Circulating leptin and adiponectin concentrations during tumor necrosis factor blockade in patients with active rheumatoid arthritis," Journal of Rheumatology, vol. 36, no. 4, pp. 724-730, 2009.

[24] M. A. Gonzalez-Gay, J. Llorca, M. T. Garcia-Unzueta et al., "High-grade inflammation, circulating adiponectin concentrations and cardiovascular risk factors in severe rheumatoid arthritis," Clinical and Experimental Rheumatology, vol. 26, no. 4, pp. 596-603, 2008.

[25] P. H. Dessein, A. J. Woodiwiss, G. R. Norton, L. Tsang, and A. Solomon, "Independent associations of total and high molecular weight adiponectin with cardiometabolic risk and surrogate markers of enhanced early atherogenesis in black and white patients with rheumatoid arthritis: a cross-sectional study, Arthritis Research \& Therapy, vol. 15, no. 5, article R128, 2013.

[26] M. A. Gonzalez-Gay, C. Gonzalez-Juanatey, L. RodriguezRodriguez, J. A. Miranda-Filloy, J. Martin, and J. Llorca, "Lack of association between adipokines and ghrelin and carotid intimamedia thickness in patients with severe rheumatoid arthritis," Clinical and Experimental Rheumatology, vol. 29, no. 2, pp. 358359, 2011.

[27] Y. H. Rho, C. P. Chung, J. F. Solus et al., "Adipocytokines, insulin resistance, and coronary atherosclerosis in rheumatoid arthritis," Arthritis and Rheumatism, vol. 62, no. 5, pp. 1259$1264,2010$.

[28] P. H. Dessein, A. J. Woodiwiss, G. R. Norton, and A. Solomon, "Rheumatoid arthritis is associated with reduced adiposity but not with unfavorable major cardiovascular risk factor profiles and enhanced carotid atherosclerosis in black Africans from a developing population: a cross-sectional study," Arthritis Research \& Therapy, vol. 15, no. 4, article R96, 2013.

[29] A. Simon, J.-L. Megnien, and G. Chironi, "The value of carotid intima-media thickness for predicting cardiovascular risk," Arteriosclerosis, Thrombosis, and Vascular Biology, vol. 30, no. 2, pp. 182-185, 2010.

[30] S. A. Riccio, A. A. House, J. D. Spence, A. Fenster, and G. Parraga, "Carotid ultrasound phenotypes in vulnerable populations," Cardiovascular Ultrasound, vol. 4, article 44, 2006. 
[31] S. H. Johnsen, E. B. Mathiesen, O. Joakimsen et al., "Carotid atherosclerosis is a stronger predictor of myocardial infarction in women than in men: a 6-year follow-up study of 6226 persons: the Tromsø study," Stroke, vol. 38, no. 11, pp. 2873-2880, 2007.

[32] S. Ebrahim, O. Papacosta, P. Whincup et al., "Carotid plaque, intima media thickness, cardiovascular risk factors, and prevalent cardiovascular disease in men and women: the British regional heart study," Stroke, vol. 30, no. 4, pp. 841-850, 1999.

[33] J. D. Spence and R. A. Hegele, "Noninvasive phenotypes of atherosclerosis," Arteriosclerosis, Thrombosis, and Vascular Biology, vol. 24, no. 11, pp. e188-e188, 2004.

[34] S. H. Johnsen and E. B. Mathiesen, "Carotid plaque compared with intima-media thickness as a predictor of coronary and cerebrovascular disease," Current Cardiology Reports, vol. 11, no. 1, pp. 21-27, 2009.

[35] J. L. Rourke, H. J. Dranse, and C. J. Sinal, “Towards an integrative approach to understanding the role of chemerin in human health and disease," Obesity Reviews, vol. 14, no. 3, pp. 245-262, 2013.

[36] P. H. Dessein, G. R. Norton, A. J. Woodiwiss, and A. Solomon, "Independent relationship between circulating resistin concentrations and endothelial activation in rheumatoid arthritis," Annals of the Rheumatic Diseases, vol. 72, no. 9, pp. 1586-1588, 2013.

[37] P. H. Dessein and A. Solomon, "Towards the elucidation of the true impact of adipocytokines on cardiovascular risk in rheumatoid arthritis," Arthritis Research \& Therapy, vol. 14, no. 127, 2013.

[38] P. H. Dessein, L. Tsang, G. R. Norton, A. J. Woodiwiss, and A. Solomon, "Retinol binding protein 4 concentrations relate to enhanced atherosclerosis in obese patients with rheumatoid arthritis," PLoS ONE, 2014.

[39] P. H. Dessein, G. R. Norton, A. J. Woodiwiss, and A. Solomon, "Age impacts on the independent relationships of leptin with cardiometabolic risk and surrogate markers of enhanced early atherogenesis in black and white patients with rheumatoid arthritis: a cross-sectional study," Rheumatology International, vol. 34, no. 3, pp. 329-339, 2014.

[40] F. C. Arnett, S. M. Edworthy, D. A. Bloch et al., "The American Rheumatism Association 1987 revised criteria for the classification of rheumatoid arthritis," Arthritis and Rheumatism, vol. 31, no. 3, pp. 315-324, 1988.

[41] D. Aletaha, T. Neogi, and A. J. Silman, "2010 rheumatoid arthritis classification criteria: an American College of Rheumatology/European League against Rheumatism collaborative initiative," Annals of the Rheumatic Diseases, vol. 69, no. 10, p. 1892, 2010.

[42] A. Gülfe, D. Aletaha, T. Saxne, and P. Geborek, "Disease activity level, remission and response in established rheumatoid arthritis: performance of various criteria sets in an observational cohort, treated with anti-TNF agents," BMC Musculoskeletal Disorders, vol. 10, article 41, 2009.

[43] E. Myasoedova, C. S. Crowson, C. Turesson, S. E. Gabriel, and E. L. Matteson, "Incidence of extraarticular rheumatoid arthritis in olmsted county, Minnesota, in 1995-2007 versus 1985-1994: a population-based study," Journal of Rheumatology, vol. 38, no. 6, pp. 983-989, 2011.

[44] A. S. Levey, J. Coresh, E. Balk, A. T. Kausz, A. Levin, and M. W. Steffes, "National Kidney Foundation practice guidelines for chronic kidney disease: evaluation, classification, and stratification," Annals of Internal Medicine, vol. 139, no. 7, pp. 137-147, 2003.

[45] P. H. Dessein, B. F. Christian, and A. Solomon, "Which are the determinants of dyslipidemia in rheumatoid arthritis and does socioeconomic status matter in this context?" Journal of Rheumatology, vol. 36, no. 7, pp. 1357-1361, 2009.

[46] A. D. Gepner, C. E. Korcarz, S. E. Aeschlimann et al., "Validation of a carotid intima-media thickness border detection program for use in an office setting," Journal of the American Society of Echocardiography, vol. 19, no. 2, pp. 223-228, 2006.

[47] P. H. Dessein, B. I. Joffe, M. G. Veller et al., "Traditional and nontraditional cardiovascular risk factors are associated with atherosclerosis in rheumatoid arthritis," Journal of Rheumatology, vol. 32, no. 3, pp. 435-442, 2005.

[48] P.-J. Touboul, M. G. Hennerici, S. Meairs et al., "Mannheim carotid intima-media thickness consensus (2004-2006). An update on behalf of the Advisory Board of the 3rd and 4th Watching the Risk Symposium, 13th and 15th European Stroke Conferences, Mannheim, Germany, 2004, and Brussels, Belgium, 2006," Cerebrovascular Diseases, vol. 23, no. 1, pp. 7580, 2007.

[49] P. H. Dessein, M. Tobias, and M. G. Veller, "Metabolic syndrome and subclinical atherosclerosis in rheumatoid arthritis," Journal of Rheumatology, vol. 33, no. 12, pp. 2425-2432, 2006.

[50] J. T. Giles, M. Allison, C. O. Bingham III, W. M. Scott Jr., and J. M. Bathon, "Adiponectin is a mediator of the inverse association of adiposity with radiographic damage in rheumatoid arthritis," Arthritis Care and Research, vol. 61, no. 9, pp. 1248-1256, 2009.

[51] M. R. Evans, A. Escalante, D. F. Battafarano, G. L. Freeman, D. H. O'Leary, and I. del Rincõn, "Carotid atherosclerosis predicts incident acute coronary syndromes in rheumatoid arthritis," Arthritis and Rheumatism, vol. 63, no. 5, pp. 1211-1220, 2011.

[52] P. H. Dessein, R. Lopez-Mejias, and C. Gonzalez-Juanatey, "Independent relationship of osteoprotegerin concentrations with endothelial activation and carotid atherosclerosis in patients with severe rheumatoid arthritis," The Journal of Rheumatology, vol. 41, no. 3, pp. 429-436, 2014.

[53] H. M. Kremers, P. J. Nicola, C. S. Crowson, K. V. Ballman, and S. E. Gabriel, "Prognostic importance of low body mass index in relation to cardiovascular mortality in rheumatoid arthritis," Arthritis and Rheumatism, vol. 50, no. 11, pp. 3450-3457, 2004.

[54] A. Escalante, R. W. Haas, and I. del Rincón, "Paradoxical effect of body mass index on survival in rheumatoid arthritis: role of comorbidity and systemic inflammation," Archives of Internal Medicine, vol. 165, no. 14, pp. 1624-1629, 2005.

[55] P. H. Dessein, A. Solomon, A. J. Woodiwiss, G. R. Norton, L. Tsang, and M. A. Gonzalez-Gay, "Marked independent relationship between circulating interleukin- 6 concentrations and endothelial activation in rheumatoid arthritis," Mediators of Inflammation, vol. 2013, Article ID 510243, 10 pages, 2013.

[56] N. Sattar and S. M. Nelson, "Adiponectin, diabetes, and coronary heart disease in older persons: unraveling the paradox," Journal of Clinical Endocrinology and Metabolism, vol. 93, no. 9, pp. 3299-3301, 2008.

[57] M. A. Gonzalez-Gay, C. Gonzalez-Juanatey, A. Piñeiro, C. Garcia-Porrua, A. Testa, and J. Llorca, "High-grade C-reactive protein elevation correlates with accelerated atherogenesis in patients with rheumatoid arthritis," Journal of Rheumatology, vol. 32, no. 7, pp. 1219-1223, 2005. 
[58] M. A. Gonzalez-Gay, C. Gonzalez-Juanatey, M. J. Lopez-Diaz et al., "HLA-DRB1 and persistent chronic inflammation contribute to cardiovascular events and cardiovascular mortality in patients with rheumatoid arthritis," Arthritis Care and Research, vol. 57, no. 1, pp. 125-132, 2007.

[59] M. A. Gonzalez-Gay, M. T. Garcia-Unzueta, C. GonzalezJuanatey et al., "Anti-TNF- $\alpha$ therapy modulates resistin in patients with rheumatoid arthritis," Clinical and Experimental Rheumatology, vol. 26, no. 2, pp. 311-316, 2008.

[60] A. Koskinen, S. Juslin, R. Nieminen, T. Moilanen, K. Vuolteenaho, and E. Moilanen, "Adiponectin associates with markers of cartilage degradation in osteoarthritis and induces production of proinflammatory and catabolic factors through mitogen-activated protein kinase pathways," Arthritis Research and Therapy, vol. 13, no. 6, article R184, 2011. 


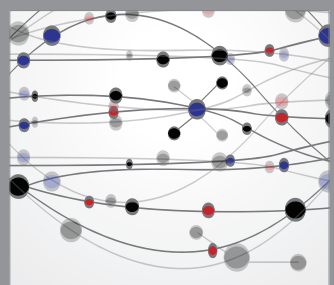

The Scientific World Journal
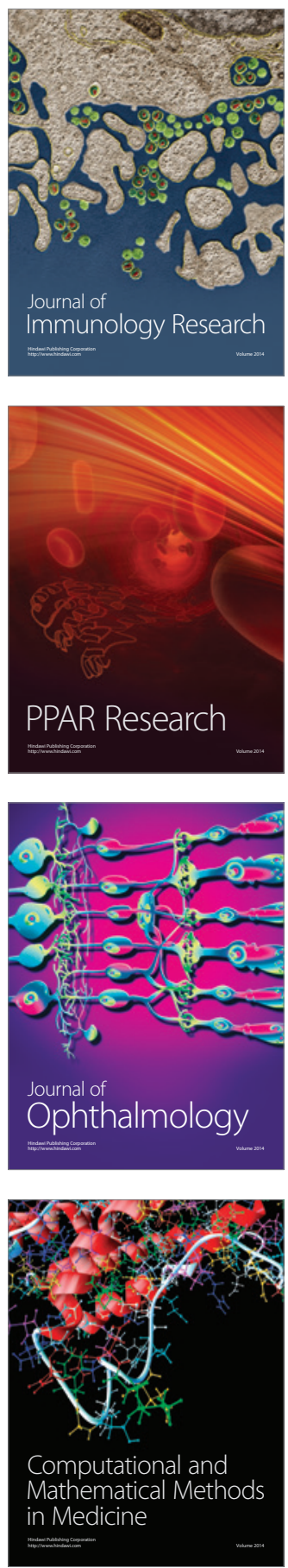

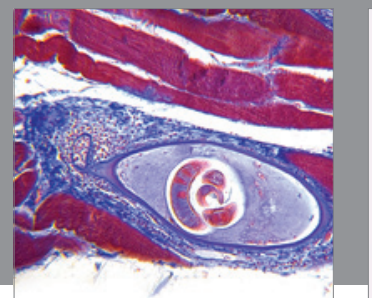

Gastroenterology

Research and Practice
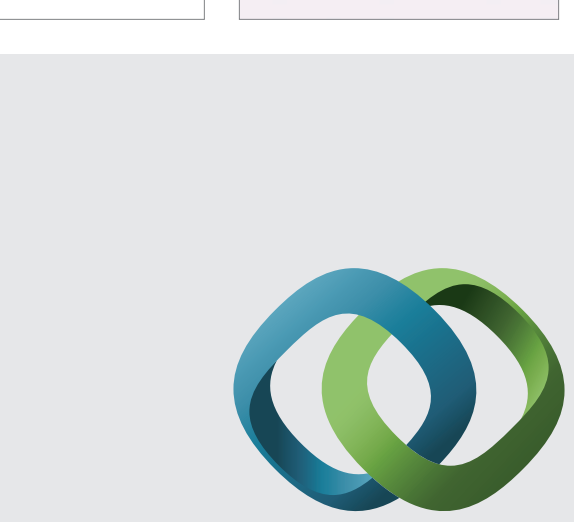

\section{Hindawi}

Submit your manuscripts at

http://www.hindawi.com
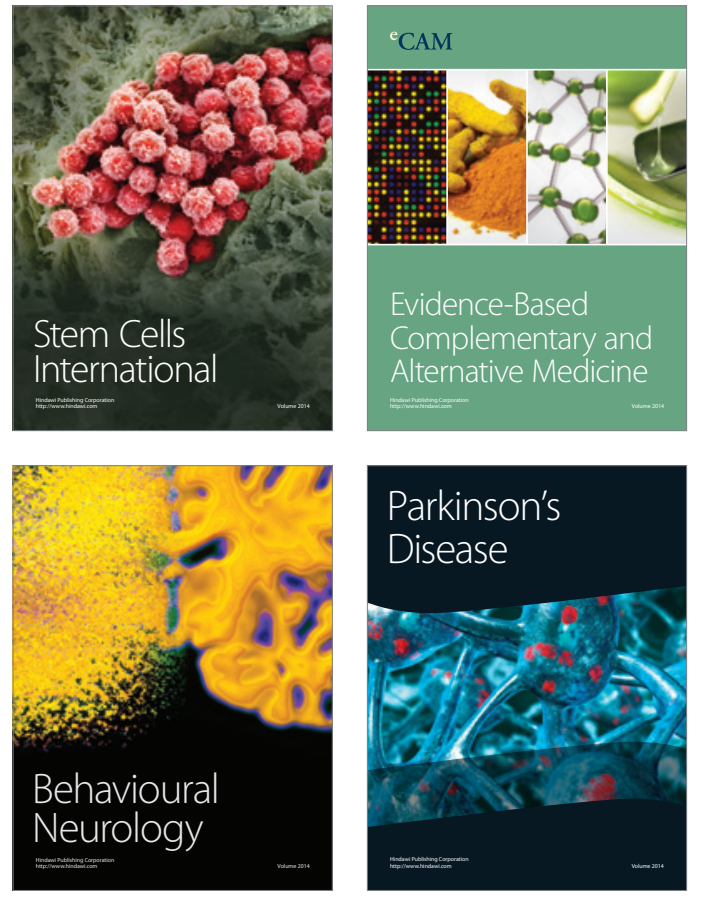
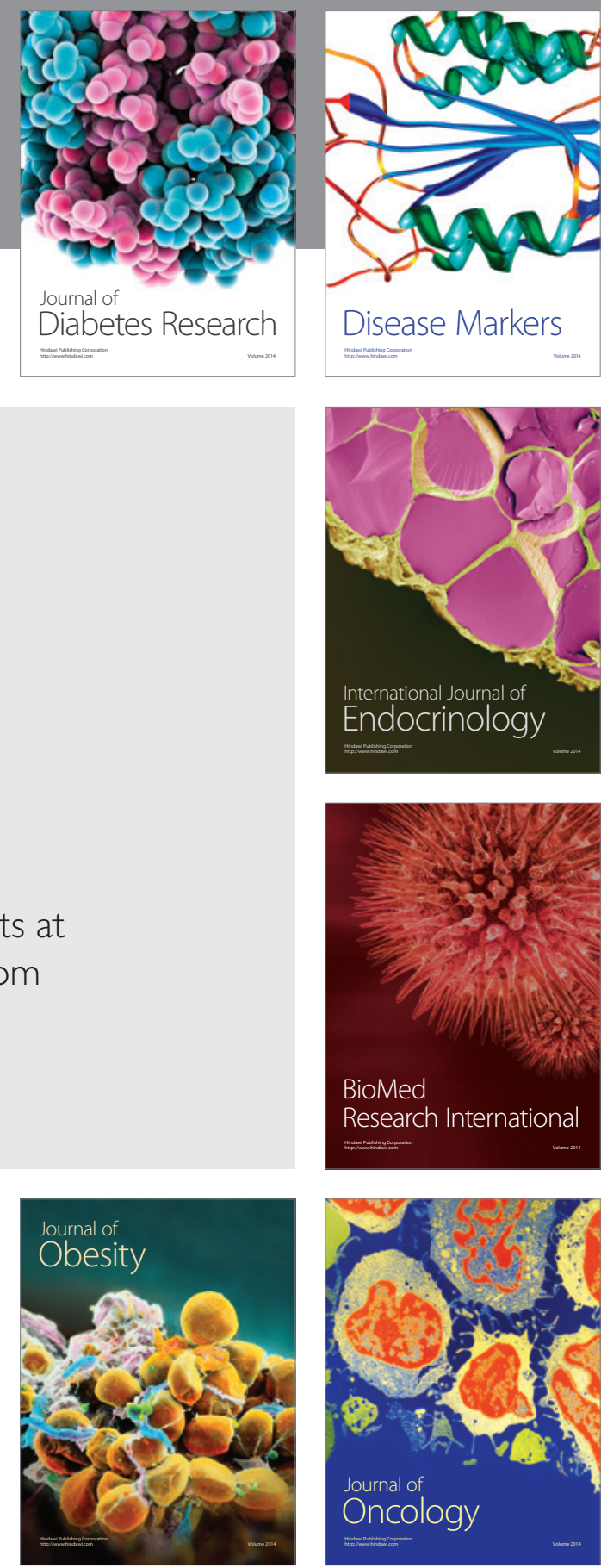

Disease Markers
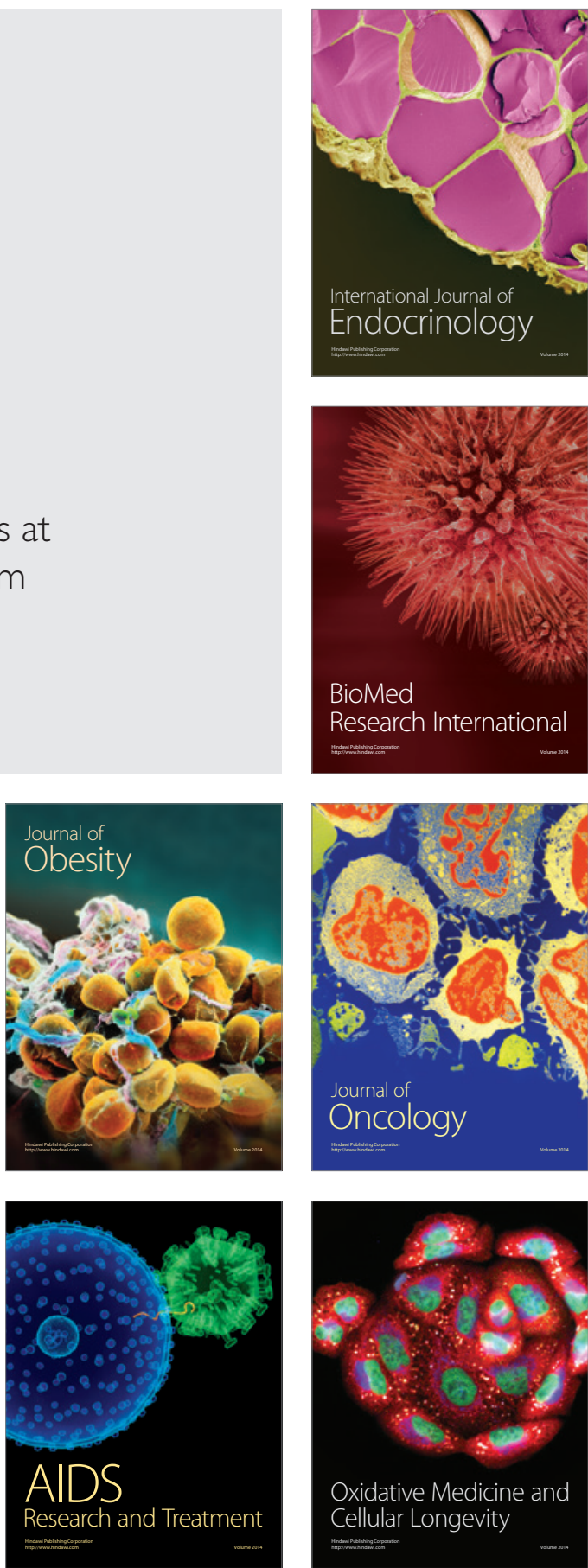